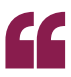

$P$. aeruginosa inhabiting different regions of the same lung evolved divergently over many years קפ

\title{
BACTERIAL EVOLUTION
}

\section{To divide and conquer}

Pseudomonas aeruginosa is the predominant pathogen in cystic fibrosis (CF) lung disease, and a single lineage can persist within a patient for life. It is known that $P$. aeruginosa evolves within the lungs; however, the mechanisms that underlie this process have been unclear. Now, Jorth et al. report that the genetic diversification of $P$. aeruginosa within the lungs of patients with CF is driven by regional isolation.

The authors sampled the upper, middle and lower lobe airways of ten pairs of CF lungs that were removed during transplantation. Using wholegenome and 16S rRNA sequencing, and random amplification of polymorphic DNA (RAPD) analysis, the authors determined that most bacteria infecting the lungs were $P$. aeruginosa and, in each patient, these belonged to a single lineage.

Next, the authors tested isolates for heritable phenotypes, including motility and antibiotic resistance, and found that most of these phenotypes were expressed by different proportions of bacteria in different lung regions. By classifying isolates into subpopulations based on multiple phenotypes, the authors determined that regional populations of $P$. aeruginosa are distinct.

To further assess regional $P$. aeruginosa populations, the authors carried out proteomic analysis of pooled isolates from each region of three lung pairs. Protein expression profiles were similar in isolates from the same region but differed significantly between regional populations, suggesting that $P$. aeruginosa evolves independently in different regions of the lung.
Consistent with this idea, bacteria isolated from the same region tended to cluster together in phylogenetic analyses of whole-genome sequencing data. Together, these results indicated that $P$. aeruginosa inhabiting different regions of the same lung evolved divergently over many years and that mixing within the lung is limited.

As CF disease tends to be more severe in the upper lung than in the lower lung, the authors looked for characteristics associated with these regions. Although phenotype, protein expression and genetic mutations differed significantly between regions within individual lungs, the patterns of regional differences were not consistent between lungs.

Finally, assessment of functional differences within a single lung revealed that the dominant subpopulations infecting the upper and lower lobes differed significantly in their growth capacity, stress tolerance, antibiotic susceptibility, cytotoxicity and virulence in a mouse lung infection model, suggesting that selective pressures differ by region.

The authors conclude that isolation in different lung regions drives divergent evolution of $P$. aeruginosa, giving rise to phenotypically specialized variants that might facilitate bacterial adaptation under changing conditions, and suggest that similar diversification mechanisms could occur in other types of chronic infections.

\section{Denise Waldron}

